\title{
Perspective The Manifest Destiny of Cocaine Research
}

\author{
Peter W Kalivas*, I \\ 'Department of Neurosciences, Medical University of South Carolina, Charleston, SC, USA
}

Neuropsychopharmacology (2009) 34, 1089-1090; doi: 10. 1038/npp.2009.9; published online 4 February 2009

In this issue of Neuropsychopharmacology there are nine articles describing important discoveries that advance our understanding of the neurobiology of cocaine addiction. That such a confluence of unsolicited research would appear in a single issue of a major journal is surprising, but even more remarkable is that after perusing this issue's compendium of cocaine-related research it is apparent how thoroughly the study of cocaine addiction infiltrates the field of neurobiology. The growth of research associated with cocaine addiction has been exponential (52 publications in 1978, 768 publications in 2008, PubMed search). Importantly, this research has evolved from work based largely on the behavioral and dopaminergic pharmacology of cocaine into diverse neurobiological mechanisms in areas such as learning and memory, neuroplasticity, and genetic and epigenetic regulation of behavior. This expansion is driven largely by the fact that focusing on dopaminergic reward mechanisms has not yielded an effective therapy for cocaine addiction, and a realization that effective therapies must consider chronic cocaine-induced neuroplasticity that underpins synaptic and circuit efficiency, and fundamental adaptive behavioral processes such as learning. The evolutionary chronology of cocaine research is well manifested in the papers in this issue.

Three of the papers further explore involvement of dopamine transmission in the acute and chronic effects of cocaine. Using a naturally occurring mouse line that lacks a nigrostriatal dopamine system, but appears to fully express mesoaccumbens dopamine neurons, Beeler et al find that in contrast to convention, the nigrostriatal system is critical for both the acute and sensitized locomotor effects of cocaine. The studies by Oleson et al and Beveridge et al examine upregulation of dopamine transporters (DAT) induced by chronic cocaine, the former describing changes in DAT during cocaine use, and the latter makes the surprising discovery using an elegant primate model that the upregulation in DAT is short lived and absent by 90

\footnotetext{
*Correspondence: Dr PW Kalivas, Department of Neurosciences, Medical University of South Carolina, 167 Ashley Avenue, Suite 607, PO Box 250677, 173 Ashley Ave, Charleston, SC 29425, USA, Tel: + I 843792 4424, Fax: + I 843792 4423,

E-mail: kalivasp@musc.edu

Received 8 January 2009; accepted 9 January 2009
}

days of cocaine withdrawal. Together, these papers further catalogue DAT involvement in drug intake and withdrawal.

In part driven by the field's need to characterize novel pharmacotherapeutic targets, the other six cocaine papers in this issue leave the focus on dopamine transmission behind. Two papers explore cocaine-induced signaling events beyond dopamine receptor stimulation. It has become clear that stress and anxiety are critical initiators in relapse (Koob and Kreek, 2007), and Rudoy et al elaborate on the established role of corticotropin-releasing factor in anxiety by showing that the ability of $\beta$-adrenergic transmission to modulate withdrawal anxiety involves regulation of CRF synthesis. Garcia-Fuster et al engage a novel tact by exploring cocaine regulation of Fas-associated protein with death domain (FADD), in part because FADD is an activator of intracellular proapoptotic events (Park et al, 2007). Acute cocaine-induced FADD upregulation poses FADD as an early signaling event in developing addiction; a notion they further support by showing that rats bred for high behavioral responding to cocaine have elevated FADD.

The use of selective breeding by Garcia-Fuster et al highlights the importance of identifying the genes that confer vulnerability to developing addiction. It is generally believed that the genes associated with vulnerability to addiction also serve to regulate other behaviors (Edenberg and Kranzler, 2005), a notion highlighted by Fattore et al who show that animals bred for rapid acquisition of avoidance in a shuttle-box task show greater acquisition and maintenance of cocaine self-administration, as well as reinstatement of cocaine-seeking. Taking the concept of genetic vulnerability into human addicts, Yuferov et al identify associations between cocaine addicts and single nucleotide polymorphisms (SNPs) of the prodynorphin gene. Importantly, expression of these SNPs was associated with reduced preprodynorphin mRNA in post-mortem striatal tissue from cocaine addicts, reinforcing the proposition that manipulating dynorphin transmission may be ameliorative in cocaine addiction (Shippenberg et al, 2007). In addition to genetic vulnerabilities, epigenetic regulation of gene expression may confer vulnerability to addiction (Renthal and Nestler, 2008), and Solinas et al demonstrate that environmental enrichment reduces the induction of cocaine-induced behavioral plasticity (eg sensitization). 
Although this was not associated with altered cocaineinduced dopamine release, associations were made with transcriptional regulators such as zif-268 and Delta-Fos B.

Finally, while rewarding mechanisms regulate the development of addiction, recent studies indicate that cognitive deficits produced by chronic cocaine use may help sustain addictive behavior (Rogers and Robbins, 2001); a possibility supported by Woicik et al showing that addicts currently using cocaine have improved cognitive functioning relative to abstinent cocaine abusers. This raises the prospect of cognitive enhancers that mimic cocaine-induced restoration of cognitive function being an effective substitution pharmacotherapy.

The nine cocaine research endeavors described in this issue exemplify an ongoing discovery process in the field of cocaine addiction that is expanding into virtually all aspects of neuroscience. The need to harvest novel mechanisms is manifested by the difficulty we have had transforming our current understanding of the neurobiology of addiction into effective pharmacotherapies.

\section{DISCLOSURE/CONFLICT OF INTEREST}

The author declares no conflict of interest.

\section{REFERENCES}

Edenberg HJ, Kranzler HR (2005). The contribution of genetics to addiction therapy approaches. Pharmacol Ther 108: 86-93.

Koob G, Kreek MJ (2007). Stress, dysregulation of drug reward pathways, and the transition to drug dependence. Am J Psychiatry 164: 1149-1159.

Park HH, Lo YC, Lin SC, Wang L, Yang JK, Wu H (2007). The death domain superfamily in intracellular signaling of apoptosis and inflammation. Annu Rev Immunol 25: 561-586.

Renthal W, Nestler EJ (2008). Epigenetic mechanisms in drug addiction. Trends Mol Med 14: 341-350.

Rogers RD, Robbins TW (2001). Investigating the neurocognitive deficits associated with chronic drug misuse. Curr Opin Neurobiol 11: 250-257.

Shippenberg TS, Zapata A, Chefer VI (2007). Dynorphin and the pathophysiology of drug addiction. Pharmacol Ther 116: 306-321. 